\title{
Expression and effects of leukemia inhibitory factor on nucleus pulposus degeneration
}

\author{
QIANG XIAO $^{1,2^{*}}$, JI-HUAN ZENG $^{2 *}$, HAO ZHOU $^{3 *}$, QUAN-HE QIU $^{4}$,

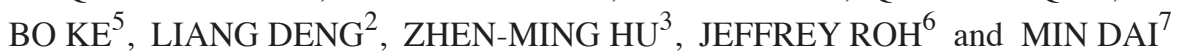 \\ ${ }^{1}$ Jiangxi Medical College, Nanchang University; ${ }^{2}$ Department of Orthopaedics, Jiangxi Provincial People's Hospital, \\ Nanchang, Jiangxi 330006; ${ }^{3}$ Department of Orthopedics, The First Affiliated Hospital of Chongqing Medical University, \\ Chongqing 400016; ${ }^{4}$ Department of Orthopaedics, The Affiliated Hospital of Jiangxi University of \\ Traditional Chinese Medicine, Nanchang, Jiangxi 330029; ${ }^{5}$ Department of Hematopathology, \\ Jiangxi Provincial People's Hospital, Nanchang, Jiangxi 330006, P.R. China; ${ }^{6}$ Swedish Neuroscience Institute, \\ Swedish Medical Center, Seattle, WA 98122, USA; ${ }^{7}$ Department of Orthopaedics, \\ The First Affiliated Hospital of Nanchang University, Nanchang, Jiangxi 330006, P.R. China
}

Received May 24, 2018; Accepted December 14, 2018

DOI: $10.3892 / \mathrm{mmr} .2019 .9874$

\begin{abstract}
Leukemia inhibitory factor (LIF) is a multifunctional cytokine. The present study aimed to determine the expression and effects of LIF on nucleus pulposus generation. Degenerated nucleus pulposus samples were obtained from animal models and patients with lumbar intervertebral disc herniation. Degradation scores of intervertebral discs were evaluated via magnetic resonance imaging (MRI) and histology, and the protein expression levels of LIF were detected. Furthermore, cultured primary human degenerated nucleus pulposus cells (DNPCs) were stimulated with various concentrations of recombinant human LIF protein (rhLIF), and aggrecan and collagen type II $\alpha 1$ (COL2 21$)$ protein expression levels were detected by western blotting. In addition, aggrecan expression was determined by toluidine blue staining. The effects of rhLIF on proliferation and apoptosis of DNPCs were evaluated by Cell Counting Kit-8 and flow cytometry, respectively. The results revealed that the degradation scores of intervertebral discs were significantly associated with modeling time, as determined by MRI and histology. In addition, the protein expression levels of LIF were initially increased in patients with lumbar disc herniation and in rabbit models, particularly in the 2 -week modeling group; however, its expression decreased with the progression of
\end{abstract}

Correspondence to: Dr Min Dai, Department of Orthopaedics, The First Affiliated Hospital of Nanchang University, 17 Yongwai Main Street, Nanchang, Jiangxi 330006, P.R. China

E-mail: daiminndyfy@163.com

${ }^{*}$ Contributed equally

Key words: leukemia inhibitory factor, nucleus pulposus degeneration, extracellular matrix components, apoptosis disc degeneration. Notably, LIF expression in each modeling group was higher than that in the control and 0 week modeling group. The in vitro study revealed that the protein expression levels of aggrecan and COL $2 \alpha 1$ were significantly increased in response to $\mathrm{rhLIF}$, in a dose-dependent manner, and statistical differences were identified between the treatment groups and control group. The results of toluidine blue staining were consistent with this finding. Although rhLIF had no effect on proliferation, it inhibited apoptosis of DNPCs in a concentration-dependent manner. In conclusion, LIF was upregulated during the process of intervertebral disc degeneration, and may promote the expression of extracellular matrix components. It may also be hypothesized that LIF acts as a potential protective factor by inhibiting apoptosis of DNPCs without affecting cell proliferation.

\section{Introduction}

Lower back pain caused by degenerative spinal conditions is a serious public health problem. It is estimated that between 2.8 and $5 \%$ of visits to health-care professionals in the United States are due to this problem, and considering direct and indirect costs, the overall cost is $>\$ 100$ billion per year (1). It has been suggested that intervertebral disc degeneration (IDD) is a major causative factor in the development of spinal degenerative diseases (2); however, the specific pathogenesis of IDD remains unclear. Extracellular matrix synthesis and catabolic imbalance are believed to be direct causes of IDD (3). It is well known that inflammatory cytokines, including interleukin IL-1, tumor necrosis factor- $\alpha$ and IL-6, serve an important role in cellular apoptosis and extracellular matrix metabolism in the process of IDD $(4,5)$.

Leukemia inhibitory factor (LIF) was first reported in a study regarding myeloid leukemia M1 cells (6). It is a 180-amino acid glycoprotein that belongs to the IL-6 sub-family, which is comprised of seven members, including IL-6, IL-11, IL-27, oncostatin M (OSM), ciliary neurotrophic factor, 
cardiotrophin-1 and cardiotrophin-like cytokine/cytokine-like factor (7). The aforementioned cytokines share the same receptors, including LIF receptor $\beta$ (LIFR $\beta$ ) and glycoprotein 130 (gp130) (8). LIF is a multifunctional cytokine that exerts various activities on numerous systems, including bone remodeling, and the nervous, muscular, endocrine and reproductive systems (9). Previous studies have reported that LIF is involved in several osteoarticular pathological conditions (10-12). Lotz et al demonstrated that LIF promotes cartilage catabolism and contributes to the pathogenesis of arthritis (13). Although chondrocytes and nucleus pulposus cells have been reported to share many cellular phenotypes (14), whether LIF is positively expressed in the nucleus pulposus of intervertebral discs, and its roles in IDD, have yet to be evaluated.

The present study aimed to evaluate the expression of LIF in the degenerative nucleus pulposus of an animal model and in patients with lumbar disc herniation. Furthermore, the effects of LIF on extracellular matrix synthesis, proliferation and apoptosis were detected in degenerative nucleus pulposus cells (DNPCs).

\section{Materials and methods}

Materials. A total of 50 adult male New Zealand rabbits (weight, $2.5 \pm 0.2 \mathrm{~kg}$ ), were purchased from the Experimental Animal Center of Chongqing Medical University (Chongqing, China). Animals were maintained in a $26^{\circ} \mathrm{C}$ room with $40-70 \%$ humidity and a 12-h light/dark cycle, and were allowed free access to food and water. In addition, degenerative disc samples were obtained from six patients undergoing lumbar disc herniation surgery (age, 27-68 years; mean age, 48.6 years; three men and three women; Pfirrmann degeneration grading, III-IV). Nucleus pulposus tissues were also obtained from a control group (age, 17-49 years; mean age, 37.1 years, three men and two women), which consisted of young trauma patients (three cases) and adolescent patients with scoliosis (two cases). All animal and patient procedures were approved by the Ethics Committee of the First Affiliated Hospital of Chongqing Medical University (Chongqing, China). Written informed consent was obtained from adult patients and the parents of adolescent patients permitting the use of their samples and the publication of any associated data.

Reagents. Pentobarbital sodium was purchased from Merck KGaA (Darmstadt, Germany). Safranin O-Fast Green staining reagent was obtained from Beijing Leagene Biotech Co., Ltd. (Beijing, China). Dulbecco's modified Eagle's medium (DMEM)/F12 and fetal bovine serum were obtained from HyClone; GE Healthcare Life Sciences (Logan, UT, USA). Recombinant Human LIF Protein was purchased from Novus Biologicals, LLC (Littleton, CO, USA). Collagenase type II was obtained from Sigma-Aldrich; Merck KGaA. Collagen type II $\alpha 1$ (COL2 $\alpha 1)$ and LIF primary antibodies were from BIOSS (Beijing, China; bs-1068R and bs-1058R), and aggrecan primary antibody was from Abcam (Cambridge, MA, USA; ab3778). Cell Counting Kit-8 (CCK-8) was purchased from Beyotime Institute of Biotechnology (Haimen, China) and the Annexin V-phycoerythrin (PE)/propidium iodide (PI) apoptosis detection kit was obtained from BD Biosciences (Franklin Lakes, NJ, USA).
Animal grouping and model establishment. A total of $50 \mathrm{New}$ Zealand white rabbits were randomly divided into the following 5 groups, with 10 rabbits in each group: The control group, and the $0,2,4$ and 8 week groups according to modeling time. According to the method introduced by Masuda et al (15), $3 \%$ pentobarbital sodium $(30 \mathrm{mg} / \mathrm{kg})$ was injected into the marginal veins of each rabbit, intervertebral discs between L4-5, L5-6 and L6-7 were exposed via the posterior lateral approach and the annulus fibrosus was punctured using a $18 \mathrm{G}$ needle $(5 \mathrm{~mm}$ depth for $5 \mathrm{sec}$ ). The incision was then closed in layers.

Imaging evaluation. After modeling at 0, 2, 4 and 8 weeks, sagittal T2-weighted magnetic resonance imaging (MRI) scans of the lumbar spine were performed under the following parameter settings: Repeat time, 2,500 msec; echo time, $85 \mathrm{msec}$; scanning layer, 11; layer thickness, $2 \mathrm{~mm}$; matrix, 196x 196; bandwidth, 31.25; field of view, 18x18. The intervertebral discs were evaluated according to the method introduced by Masudaet al (15). Images and scoring of the discs were assessed by two experienced, independent radiologists.

Histological assessment. Rabbits were sacrificed on the day that MRI examinations were conducted, after which, discs were removed and fixed in $4 \%$ paraformaldehyde for $24 \mathrm{~h}$ at room temperature. They were then decalcified in EDTA solution for 4-5 weeks (decalcifying solution was replaced every 3-4 days). Disc tissues were embedded in paraffin, sliced into $5 \mu \mathrm{m}$ sections on the median sagittal, dewaxed in water and then stained with Safranin O-Fast Green, according to the manufacturer's protocol. Pathological assessment of degeneration was performed according to the Masuda criteria under a confocal microscope (Olympus Corporation, Tokyo, Japan) (15).

Measurement of LIF expression in intervertebral disc nucleus pulposus. Total proteins were extracted from nucleus pulposus samples harvested from rabbit and human discs by grinding in liquid nitrogen (PROTTOT-1KT, Sigma-Aldrich; Merck $\mathrm{KGaA}$ ). Protein concentrations were then measured using the bicinchoninic acid (BCA) method and the protein expression levels of LIF were detected in each group by western blot analysis. Briefly, $50 \mu \mathrm{g}$ of extracted proteins were separated by $10 \%$ SDS-PAGE, then were electro-blotted onto PVDF membranes (EMD Millipore, Billerica, MA, USA). The membranes were blocked using 5\% blocking buffer (P0252; Beyotime Institute of Biotechnology) at room temperature for $1 \mathrm{~h}$. After incubation with rabbit anti-LIF antibody (1:1,000; SAB2102317; Sigma-Aldrich; Merck KGaA), membranes were washed 3 times with TBST (P0023C; Beyotime Institute of Biotechnology) for $10 \mathrm{~min}$ and followed by incubation with a secondary antibody (1:1,000; Sigma-Aldrich; Merck $\mathrm{KGaA}$ ) for $2 \mathrm{~h}$ at room temperature, and were then washed 3 times again with TBST. Relative levels of immunoreactivity were quantified using the Kodak In-vivo Imaging System (Kodak, Rochester, NY, USA). Rabbit anti-GADPH (1:2,000; SAB2108668; Sigma-Aldrich; Merck KGaA) was used as an internal control for the concentration of proteins loaded. Images were analyzed using Image $J$ version $2 x$ (National Institutes of Health, Bethesda, MD, USA). 
Culture of human DNPCs. Human nucleus pulposus cells were isolated from the obtained degenerative discs of patients as described previously (16). Blood in nucleus pulposus samples was washed away using PBS supplemented with penicillin (800,000 U/l) and streptomycin (1,000,000 U/1). The samples were cut into pieces using ophthalmic scissors, and were then digested using $0.25 \%$ trypsin and $0.2 \%$ type II collagenase. After sieving through a 200-mesh cell sieve, the cells were centrifuged $\left(300 \times \mathrm{g}, 4^{\circ} \mathrm{C}, 5 \mathrm{~min}\right)$ and suspended in a culture flask and were cultured in DMEM/F12 medium containing $20 \%$ fetal bovine serum at $37^{\circ} \mathrm{C}$ in an incubator containing $5 \% \mathrm{CO}_{2}$.

Detection of aggrecan and COL $2 \alpha 1$ protein. Passage (P)2-P3 cells were seeded into $10-\mathrm{cm}$ culture dishes and treated with recombinant human LIF protein (rhLIF) at 0,10 , 20,50 or $100 \mathrm{ng} / \mathrm{ml}$ for $24 \mathrm{~h}$ at room temperature, or were treated with $100 \mathrm{ng} / \mathrm{ml} \mathrm{rhLIF}$ for $0,24,48$ or $72 \mathrm{~h}$, as described previously (17). Subsequently, cells from each group were collected to extract total protein. After protein concentration was measured using the BCA method, the protein expression levels of aggrecan and COL $2 \alpha 1$ were detected by western blot analysis.

P2 cells were also seeded onto glass slides and treated with 0 or $100 \mathrm{ng} / \mathrm{ml}$ rhLIF for $48 \mathrm{~h}$. The slides were collected, rinsed with PBS, fixed with $40 \mathrm{~g} / 1$ paraformaldehyde for $30 \mathrm{~min}$ at room temperature, and rinsed with PBS three times (5 min/wash). Subsequently, the cells were stained with $1 \mathrm{~g} / \mathrm{l}$ toluidine blue stain for $10 \mathrm{~min}$, washed with double distilled water for $1 \mathrm{~min}$, and then placed under an inverted phase contrast microscope to detect proteoglycan staining (18).

Cell proliferation assay. P2 cells were treated with various concentrations of rhLIF and then incubated with serum-free medium for $24 \mathrm{~h}$ at room temperature. Cell proliferation was measured using the CCK- 8 kit, according to the manufacturer's protocol. Cells were incubated with CCK-8 solution for $4 \mathrm{~h}$ and cell proliferation rate was assessed by measuring absorbance at $450 \mathrm{~nm}$.

Apoptosis analysis. The apoptotic rate was detected by flow cytometry using the Annexin V-PE/PI apoptosis detection kit. P2 cells were treated with various concentrations of rhLIF and then incubated with serum-free medium for $24 \mathrm{~h}$ at room temperature. Cells were then collected and suspended in binding buffer. Subsequently, cells were counted and incubated with $5 \mu \mathrm{l}$ Annexin V-PE and $5 \mu \mathrm{l}$ PI for $30 \mathrm{~min}$ at room temperature in the dark. Finally, flow cytometry was performed within $1 \mathrm{~h}$. Apoptotic cells were defined as Annexin V (+)/PI (-) and Annexin V (+)/PI (+).

Statistical analysis. All analyses were performed using SPSS version 19.0 (IBM Corp., Armonk, NY, USA). All data were presented as the means \pm standard deviation. For each independent in vitro experiment, at least three technical replicates were analyzed. Differences between groups were analyzed by one-way analysis of variance followed by Student-Newman-Keuls post hoc test to determine multiple comparisons. Correlation analysis of the MRI score, histological score and modeling time was conducted using the
Spearman Rank Correlation test. $\mathrm{P}<0.05$ was considered to indicate a statistically significant difference.

\section{Results}

Successful generation of an IDD model. Nuclei from the control group and the 0 week modeling group exhibited a uniform, high signal in MRI. However, the nuclei signals from the 2 week modeling group were slightly decreased, and the signals were further reduced in the 4 week modeling group. In the 8 week modeling group, no signal was detected in the nuclei. According to the modified Thompson scoring system, scores of the control group and the 0 week modeling group were consistent with each other, whereas scores of the 2, 4 and 8 week modeling groups increased with time. The differences among the groups were statistically significant $(1.000 \pm 0.000$, $1.000 \pm 0.200,2.000 \pm 0.471,2.900 \pm 0.568$ and $3.900 \pm 0.316$ in the control, 0, 2, 4 and 8 week groups, respectively; $\mathrm{P}<0.05$; Fig. 1).

At low magnification, the nucleus pulposus of the control group and the 0 week group (magnification, $x 40$ ) was round or oval shaped, and the annulus fibrosis was in a regular circular arrangement. At high magnification (magnification, x400), the foamy gel-like matrix was abundant and nucleated cells were clustered or had aggregated into a ring. In the 2 week modeling group, the nucleus pulposus was smaller than in the 0 week modeling group and the junction with the annulus fibrosis was unclear or irregular. In addition, mild extracellular matrix shrinkage and scattered nucleus pulposus cells were observed. In the 4 week modeling group, the nucleus pulposus exhibited obvious extracellular matrix shrinkage and fibrosis, and the number of normal nucleus pulposus cells was markedly reduced. In the 8 week modeling group, fibrosis in the nucleus pulposus was further aggravated and it was continuous with the annulus fibrosus; the junction between the two regions was obviously fractured and delaminated. According to Masuda's histological scoring, there was no significant difference between the control group and the 0 week modeling group. However, the degeneration scores increased with the prolongation of modeling time, and differences among the modeling groups were statistically significant $(4.000 \pm 0.000$, $4.000 \pm 0.0400,7.300 \pm 0.483,9.300 \pm 1.160$ and $11.800 \pm 0.422$ in the control, 0, 2, 4 and 8 week groups, respectively; $\mathrm{P}<0.05$; Fig. 2).

Degeneration scores of intervertebral discs are significantly correlated with modeling time. There was a significant positive correlation between modeling time and MRI score $(r=0.939$; $\mathrm{P}<0.05)$. In addition, the correlation between modeling time and histological score was significantly positively $(r=0.958$; $\mathrm{P}<0.05)$. MRI score was also significantly correlated with histological score $(\mathrm{r}=0.967 ; \mathrm{P}<0.05)-$. The results of this correlation analysis indicated that the annulus puncture degeneration model was successfully established, and with the extension of modeling time, degeneration of intervertebral discs was aggravated.

LIF is highly expressed in the degenerated nucleus pulposus. Western blotting was used to detect LIF protein expression (Fig. 3). The protein expression levels of LIF in nucleus 


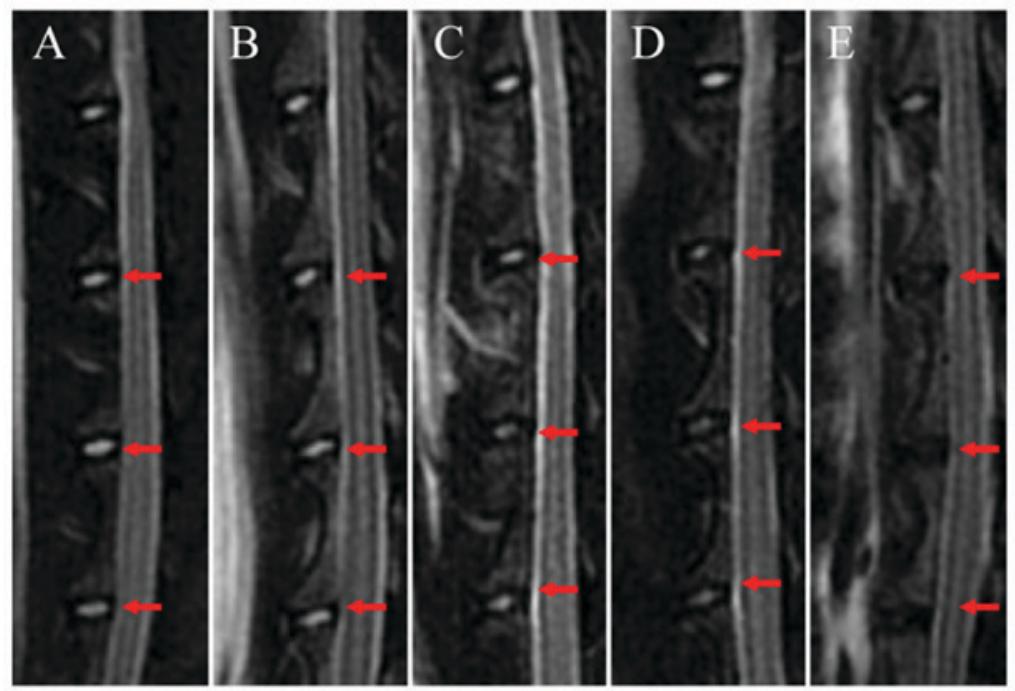

$\mathrm{F}$

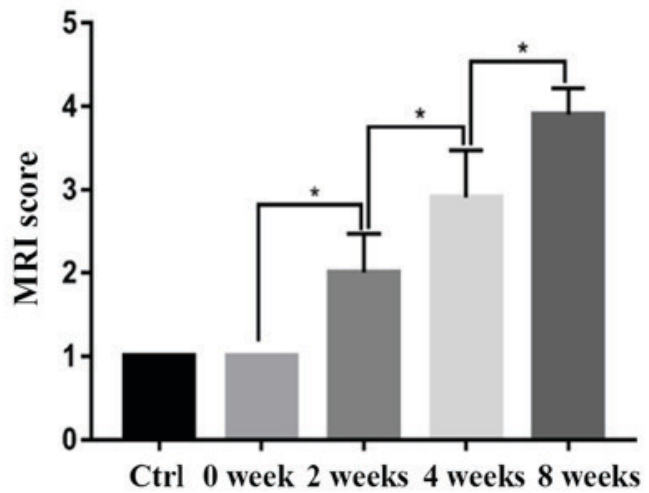

Figure 1. MRI results of each group. The intervertebral discs between L4-5, L5-6 and L6-7 were punctured with an $18 \mathrm{G}$ needle at a depth of $5 \mathrm{~mm}$ for 5 sec. (A-E) Representative MRI images of the punctured discs in the control group, and the 0,2, 4 and 8 week modeling groups, respectively. Arrows indicate punctured intervertebral discs. (F) Imaging scores of the various modeling groups. Data are presented as the means \pm standard deviation. ${ }^{*} \mathrm{P}<0.05$. MRI, magnetic resonance imaging.
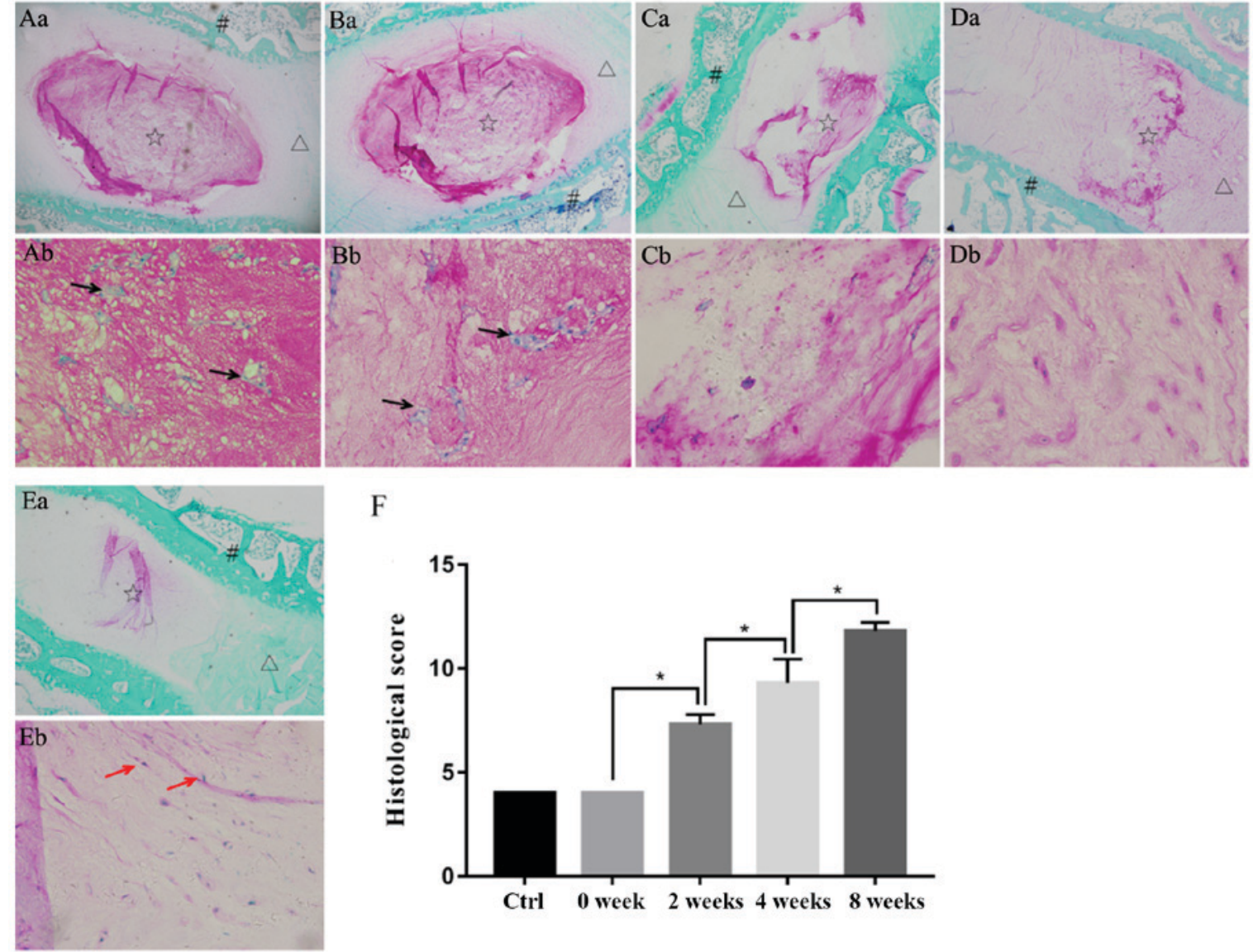

$\mathrm{F}$

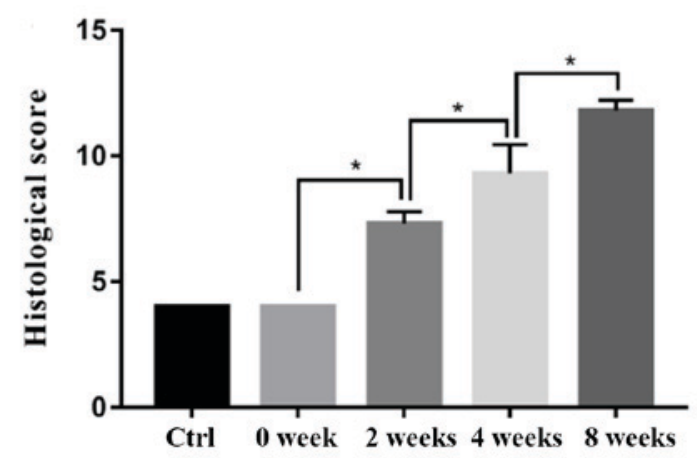

Figure 2. Safranin O-Fast Green staining results. Representative images of Safranin O-Fast Green staining of whole discs in the (Aa) control group, and the (Ba) 0, (Ca) 2, (Da) 4 and (Ea) 8 week modeling groups. Magnification, x40. Representative images of Safranin O-Fast Green staining of the nucleus pulposus in the (Ab) control group, and the (Bb) $0,(\mathrm{Cb}) 2,(\mathrm{Db}) 4$ and (Eb) 8 week modeling groups. Magnification, $\mathrm{x} 400{ }^{\star}{ }^{\star}$ indicates nucleus pulposus; ${ }^{\triangle}$ indicates annulus fibrosis; ${ }^{*}$ indicates cartilage endplate. Black arrows indicate the nucleus pulposus cells and red arrows indicate the fibroid cells. (F) Histological scores of the various modeling groups. Data are presented as the means \pm standard deviation. " $\mathrm{P}<0.05$.

pulposus samples from the control group and the 0 week modeling group were low, and the difference between them was not statistically significant $(0.205 \pm 0.147$ vs. $0.200 \pm 0.114$; $\mathrm{P}=0.459$ ). Conversely, LIF protein expression was significantly 
A

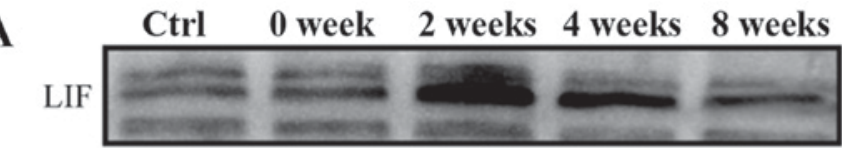

GAPDH

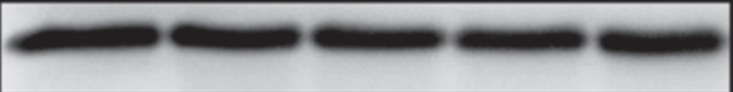

B

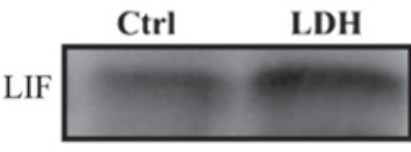

GAPDH

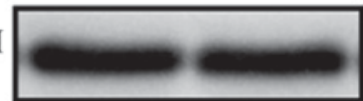

C

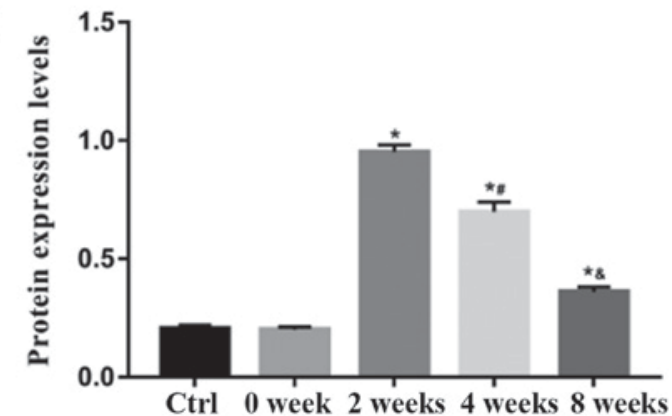

D

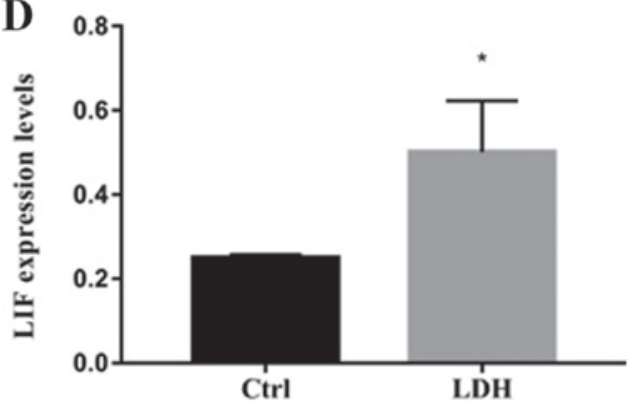

Figure 3. LIF protein expression in the nucleus pulposus. (A and C) Protein expression of LIF in rabbit nucleus pulposus samples from the control and modeling groups. ${ }^{*} \mathrm{P}<0.05$ vs. 0 week group; ${ }^{\#} \mathrm{P}<0.05$ vs. 2 week group; ${ }^{\&} \mathrm{P}<0.05$ vs. 4 week group. (B and D) Protein expression of LIF in nucleus pulposus samples from patients with LDH and the normal control group. ${ }^{*} \mathrm{P}<0.05$. Data are presented as the means \pm standard deviation. LDH, lumbar disc herniation; LIF, leukemia inhibitory factor.

higher in the 2 week modeling group; however, as the duration of modeling time and the progression of disc degeneration were prolonged, LIF protein expression was gradually decreased in the 4 and 8 week modeling groups. The differences between the $0,2,4$ and 8 week modeling groups were statistically significant $(0.200 \pm 0.114,0.950 \pm 0.301,0.698 \pm 0.410,0.360 \pm 0.210$ in the control, 0, 2, 4 and 8 week groups, respectively; $\mathrm{P}<0.05$; Fig. 3A and 3C). The protein expression levels of LIF were also significantly increased in the nucleus pulposus samples of patients with lumbar disc herniation compared with in the control group $(0.250 \pm 0.007$ vs. $0.501 \pm 0.012 ; \mathrm{P}<0.05)$ (Fig. 3B and 3D).

LIF promotes aggrecan and COL $2 \alpha 1$ protein expression in human DNPCs. Western blot analysis revealed that the protein expression levels of aggrecan and COL $2 \alpha 1$ were increased in response to increasing rhLIF concentrations (Fig. 4A-D). The protein expression levels of aggrecan and COL $2 \alpha 1$ in the rhLIF-treated groups were significantly higher compared with in the $0 \mathrm{ng} / \mathrm{ml}$ group [aggrecan: $0.210 \pm 0.007,0.304 \pm 0.023$, $0.425 \pm 0.035,0.507 \pm 0.024$ and $0.479 \pm 0.020$ in the $0,10,20$, 50 and $100 \mathrm{ng} / \mathrm{ml}$ groups, respectively $(\mathrm{P}<0.05)$; COL $2 \alpha 1$, $0.209 \pm 0.015,0.281 \pm 0.013,0.361 \pm 0.004,0.451 \pm 0.027$ and $0.489 \pm 0.028$ in the $0,10,20,50$ and $100 \mathrm{ng} / \mathrm{ml}$ groups, respectively $(\mathrm{P}<0.05)]$. At $100 \mathrm{ng} / \mathrm{ml}$, rhLIF exhibited the strongest enhancing effect on aggrecan and COL $2 \alpha$ expression, and this effect was maintained for $\geq 72 \mathrm{~h}$. The results of toluidine blue staining were consistent with those of the western blot analysis (Fig. 4E). Aggrecan was uniformly expressed in the cytoplasm in both groups; however, compared with the control group, staining intensity was markedly increased in the $100 \mathrm{ng} / \mathrm{ml}$ treatment group.

LIF inhibits apoptosis of human DNPCs. rhLIF inhibited the apoptosis of human DNPCs in a dose-dependent manner (Fig. 5A and B). As shown in Fig. 5A and B, the apoptotic rate of DNPCs was gradually decreased in response to increasing concentrations of rhLIF, and the rates were significantly reduced compared with the rate in the control group $(47.89 \pm 1.185,45.310 \pm 0.345,43.883 \pm 0.738,39.520 \pm 0.243$ and $37.493 \pm 0.340$ in the $0,10,20,50$ and $100 \mathrm{ng} / \mathrm{ml}$ groups, respectively; $\mathrm{P}<0.05)$. Conversely, rhLIF had no effect on the proliferation of human DNPCs $(0.770 \pm 0.017,0.774 \pm 0.046$, $0.785 \pm 0.027,0.765 \pm 0.037$ and $0.771 \pm 0.029$ in the $0,10,20,50$ and $100 \mathrm{ng} / \mathrm{ml}$ groups, respectively; $\mathrm{P}=0.900$ ) (Fig. 5C).

\section{Discussion}

Using the method introduced by Masuda et al (15), the present study successfully constructed an animal model of degenerative disc disease (19). A similar method of modeling was reported in a previously published study (20). Although some researchers recommend a longer modeling time of 12 weeks (21), it has been reported that the MRI signal of the discs generally reaches grade 4 degeneration at 8 weeks and typically stabilizes at later time points (22).

In vivo experiments demonstrated that in rabbits and humans, the protein expression levels of LIF were increased in degenerative nucleus pulposus compared with in the control group. Previous studies have reported that, in articular cartilage, LIF tends to act as a negative catabolic factor $(12,13)$. In a mouse model of K/BXN serum transfer arthritis, LIF knockout $\left(\mathrm{LIF}^{-/-}\right)$mice display a $>50 \%$ reduction in clinical arthritis severity. In addition, significantly lower histological cartilage scores are observed in $\mathrm{LIF}^{-/}$mice compared with in wild-type controls (23). Our previous study also suggested that LIF expression is highest in advanced articular cartilage tissue (24). In order to further clarify the role of LIF in the degeneration of intervertebral disc tissue, in vitro experiments were conducted.

Reduced synthesis of aggrecan and COL $2 \alpha 1$ proteins, and decreased numbers of nucleus pulposus cells are important 
A
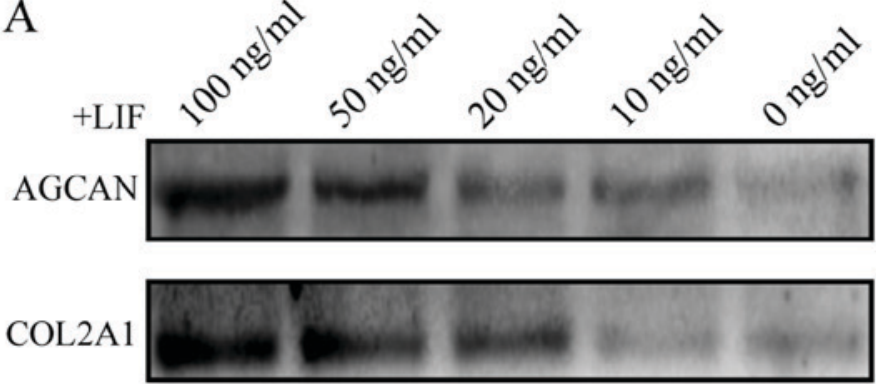

GAPDH

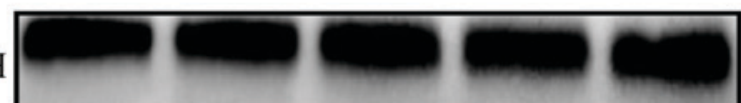

$\mathrm{C}$

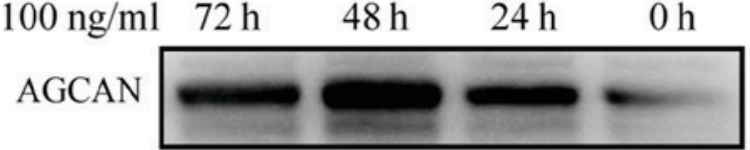

COL2A1

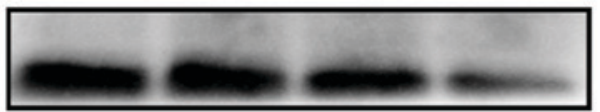

GAPDH

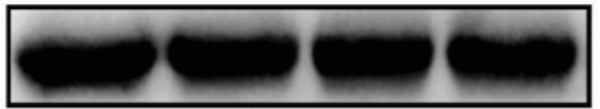

$\mathrm{E}$

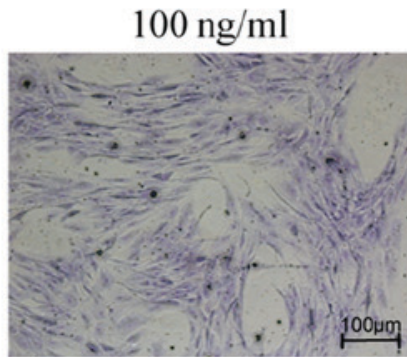

$0 \mathrm{ng} / \mathrm{ml}$

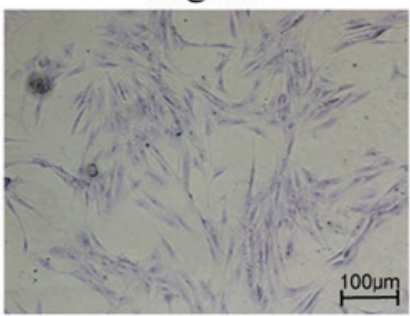

$\mathrm{B}$

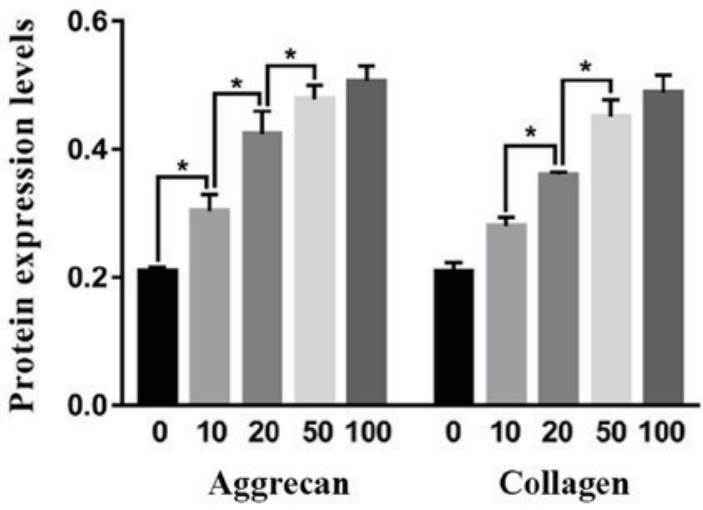

$\mathrm{D}$

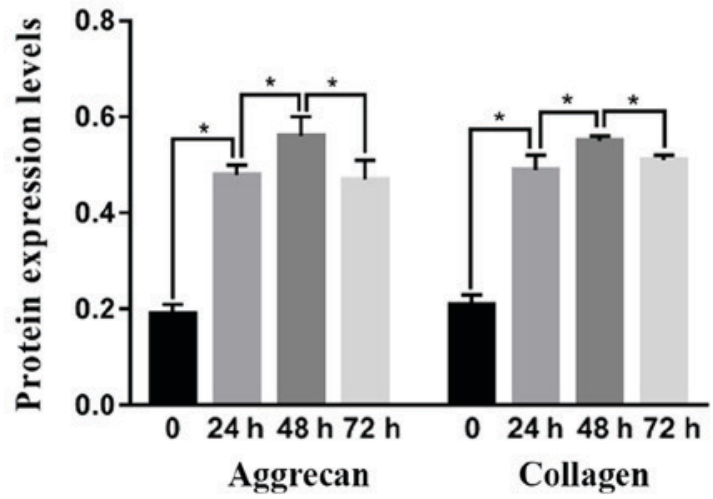

Figure 4. Protein expression of aggrecan and COL2 $\alpha 1$ in human nucleus pulposus cells stimulated with various concentrations of LIF at different durations. (A and B) Aggrecan and COL2 $\alpha 1$ protein expression in human nucleus pulposus cells treated with various concentrations of recombinant human LIF protein. (C and D) Protein expression levels of aggrecan and COL2 $\alpha 1$ in human nucleus pulposus cells treated with $100 \mathrm{ng} / \mathrm{ml}$ recombinant human LIF protein for various durations. (E) Toluidine blue staining following treatment of human nucleus pulposus cells with 0 or $100 \mathrm{ng} / \mathrm{ml}$ recombinant human LIF protein for 48 h. Data are presented as the means \pm standard deviation. " $\mathrm{P}<0.05$. COL $2 \alpha 1$, collagen type II $\alpha 1$; LIF, leukemia inhibitory factor.

features of disc degeneration $(25,26)$. Therefore, with reference to the concentration gradient set by Upadhyay et al (17), cultured primary human DNPCs were treated with various concentrations of rhLIF for different time periods and the protein expression levels of aggrecan and COL $2 \alpha 1$ were detected. The results revealed that rhLIF promoted the expression levels of both of these proteins. Toluidine blue staining, which preferentially stains proteoglycans such as aggrecan (27), confirmed the positive regulatory effect of rhLIF. Further results suggested that rhLIF had no effect on the proliferation of DNPCs, but reduced the apoptotic rate of DNPCs.

In vivo, an increase in LIF was associated with reduced breakdown of the extracellular matrix and loss of cells in the intervertebral disc tissue at a relatively early stage of degeneration (2 week modeling group). As the degeneration process progressed, the number of nucleus pulposus cells was decreased, alongside reduced LIF secretion (4 and 8 week modeling groups). Although the present study indicated that LIF may exert protective effects on the nucleus pulposus in intervertebral discs, the amount of LIF naturally secreted during the degenerative process is not sufficient to resist the progression of IDD. Therefore, administration of exogenous LIF may be able to slow degeneration to a certain extent.

LIF, IL-6 and OSM are members of the IL- 6 family. Their amino acid sequences and secondary structures are notably similar, and they have the same receptor conversion mechanism (8). Previous studies have reported that IL-6 can enhance the expression of TIMP metallopeptidase inhibitor 1 (TIMP-1) in the synovium and chondrocytes, thus blocking IL-1-mediated collagenolysis. IL-6 can also induce the production of an IL-1 receptor antagonist and thus block the 

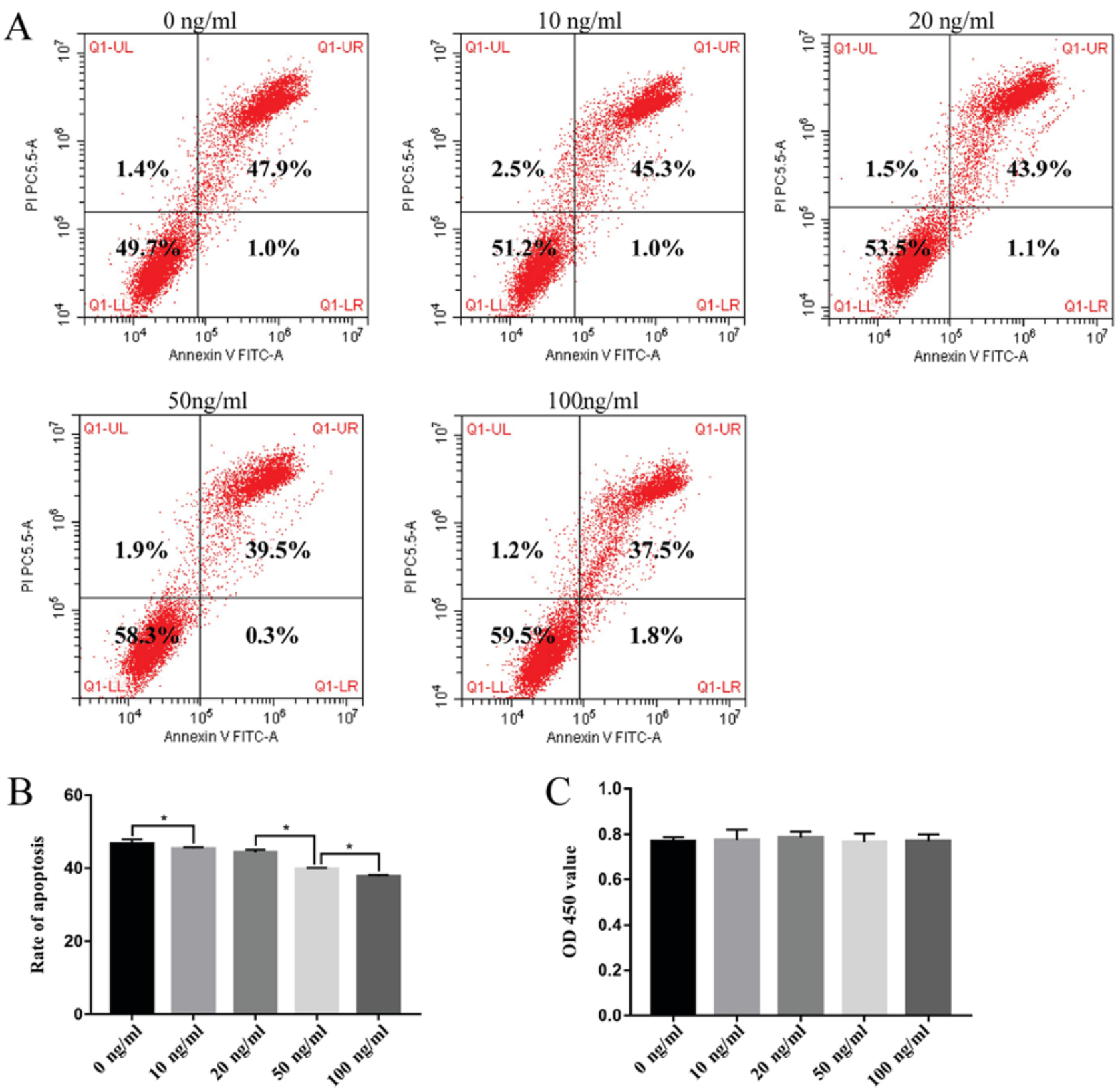

Figure 5. Effects of LIF on the proliferation and apoptosis of nucleus pulposus cells. (A and B) Apoptotic rate of human nucleus pulposus cells treated with various concentrations of recombinant human LIF. (C) Proliferation of human nucleus pulposus cells treated with various concentrations of recombinant human LIF. Data are presented as the means \pm standard deviation. ${ }^{*} \mathrm{P}<0.05$. FITC, fluorescein isothiocyanate; LIF, leukemia inhibitory factor; OD, optical density.

proinflammatory effects of IL-1 $(28,29)$. OSM significantly promotes TIMP-1 expression in chondrocytes, thus acting as a chondroprotective factor (30). Notably, Upadhyay et al reported that LIF increases the expression of TIMP-1 in chondrocytes by $\sim 4$-fold (17). The present results also indicated that LIF may exist as a protective factor in DNPCs.

The present study had several limitations. Although it was demonstrated that LIF promoted the expression of aggrecan and COL2 $\alpha 1$, and inhibited apoptosis of DNPCs in vitro, whether those effects were sustained in vivo was not investigated. Furthermore, the molecular mechanisms underlying how LIF was induced in degenerative nucleus pulposus, and how it acted as a protective factor during the process of IDD was not investigated.
LIF is a pleiotropic cytokine with a wide range of activities (31), but there is no intrinsic tyrosine kinase activity within the LIF receptor; instead, its two signaling chains, gp130 and LIFR $\beta$, are constitutively associated with members of the Janus kinase (JAK) family of tyrosine kinases (32). Therefore, future mechanistic studies may focus on JAK signaling, including the JAK/signal transducer and activator of transcription, mitogen-activated protein kinase and phosphatidylinositol 3-kinase pathways (9).

In conclusion, the present study explored the expression of LIF in degenerative intervertebral discs and discussed the protective effects of LIF on nucleus pulposus cells. LIF was upregulated during the process of IDD, and promoted the expression of extracellular matrix components. In addition, 
it was suggested that LIF may act as a potential protective factor by inhibiting apoptosis of DNPCs without affecting cell proliferation. However, to clarify the specific mechanism underlying the ability of LIF to alleviate IDD, further investigations are required.

\section{Acknowledgements}

Not applicable.

\section{Funding}

This study was supported, in part, by the National Natural Science Foundation of Jiangxi Province (grant no. 20151BAB205092) and the Key Researching and Developing Project of Jiangxi Province (grant no. 20161BBG70125).

\section{Availability of data and materials}

All data generated or analyzed during this study are included in this published article.

\section{Authors' contributions}

QX and JHZ participated in the entire process of the study. QX and HZ carried out the animal experiments. QHQ, BK and LD helped to perform the in vitro experiments, JHZ performed the statistics. ZMH and JR helped conduct the histomorphological and immunohistochemical staining, and revised the manuscript. MD designed and coordinated the study. All authors read and approved the final manuscript.

\section{Ethics approval and consent to participate}

All animal and patient procedures were approved by the Ethics Committee of the First Affiliated Hospital of Chongqing Medical University. Written informed consent was obtained from adult patients and from the parents of adolescent patients permitting the use of their samples.

\section{Patient consent for publication}

Written informed consent was obtained from adult patients and from the parents of adolescent patients permitting the publication of any associated data.

\section{Competing interests}

The authors declare that they have no competing interests.

\section{References}

1. Kepler CK, Ponnappan RK, Tannoury CA, Risbud MV and Anderson DG: The molecular basis of intervertebral disc degeneration. Spine J 13: 318-330, 2013.

2. Global Burden of Disease Study 2013 Collaborators: Global, regional, and national incidence, prevalence, and years lived with disability for 301 acute and chronic diseases and injuries in 188 countries, 1990-2013: A systematic analysis for the Global Burden of Disease Study 2013. Lancet 386: 743-800, 2015.
3. Vo N, Niedernhofer LJ, Nasto LA, Jacobs L, Robbins PD, Kang J and Evans $\mathrm{CH}$ : An overview of underlying causes and animal models for the study of age-related degenerative disorders of the spine and synovial joints. J Orthop Res 31: 831-837, 2013.

4. Risbud MV and Shapiro IM: Shapiro, role of cytokines in intervertebral disc degeneration: Pain and disc content. Nat Rev Rheumatol 10: 44-56, 2014.

5. Freemont AJ: The cellular pathobiology of the degenerate intervertebral disc and discogenic back pain. Rheumatology (Oxford) 48: 5-10, 2009.

6. Ichikawa Y: Differentiation of a cell line of myeloid leukemia. J Cell Physiol 74: 223-234, 1969.

7. Trouillas M, Saucourt C, Guillotin B, Gauthereau X, Taupin JL, Moreau JF and Boeuf H: The LIF cytokine: Towards adulthood. Eur Cytokine Netw 20: 51-62, 2009.

8. Gearing DP, Comeau MR, Friend DJ, Gimpel SD, Thut CJ, McGourty J, Brasher KK, King JA, Gillis S, Mosley B, et al: The IL-6 signal transducer, gp130: An oncostatin M receptor and affinity converter for the LIF receptor. Science 255: 1434-1437, 1992.

9. Nicola NA and Babon JJ: Leukemia inhibitory factor (LIF), Cytokine Growth Factor Rev 26: 533-544, 2015.

10. Carroll GJ and Bell MC: Leukaemia inhibitory factor stimulates proteoglycan resorption in porcine articular cartilage. Rheumatol Int 13: 5-8, 1993.

11. Naka $\mathrm{T}$ and Kishimoto T: Joint disease caused by defective gp130-mediated STAT signaling. Arthritis Res 4: 154-156, 2002.

12. Bell MC and Carroll GJ: Rheumatoid synovial fluid contains bioactive leukemia inhibitory factor with cartilage degrading activity-another target for chondroprotective intervention. J Rheumatol 27: 332-338, 2000.

13. Lotz M, Moats T and Villiger PM: Leukemia inhibitory factor is expressed in cartilage and synovium and can contribute to the pathogenesis of arthritis. J Clin Invest 90: 888-896, 1992.

14. Lee CR, Sakai D, Nakai T, Toyama K, Mochida J, Alini M and Grad S: A phenotypic comparison of intervertebral disc and articular cartilage cells in the rat. Eur Spine J 16: 2174-2185, 2007.

15. Masuda K, Aota Y, Muehleman C, Imai Y, Okuma M, Thonar EJ, Andersson GB and An HS: A novel rabbit model of mild, reproducible disc degeneration by an anulus needle puncture: correlation between the degree of disc injury and radiological and histological appearances of disc degeneration. Spine (Phila Pa 1976) 30: 5-14, 2005.

16. Zhang X, Hu Z, Hao J and Shen J: Low intensity pulsed ultrasound promotes the extracellular matrix synthesis of degenerative human nucleus pulposus cells through FAK/PI3K/Akt pathway. Spine (Phila Pa 1976) 41: E248-E254, 2016.

17. Upadhyay A, Sharma G, Kivivuori S, Raye WS, Zabihi E, Carroll GJ and Jazayeri JA: Role of a LIF antagonist in LIF and OSM induced MMP-1, MMP-3, and TIMP-1 expression by primary articular chondrocytes. Cytokine 46: 332-338, 2009.

18. Ahadiat O, Higgins S, Ly A and Wysong A: Toluidine blue stain of dermatofibrosarcoma protuberans: Highlighting its use in mohs. Dermatol Surg 43: 1496-1498, 2017.

19. Han B, Zhu K, Li FC, Xiao YX, Feng J, Shi ZL, Lin M, Wang J and Chen QX: A simple disc degeneration model induced by percutaneous needle puncture in the rat tail. Spine (Phila $\mathrm{Pa}$ 1976) 33: 1925-1934, 2008.

20. Vaudreuil N, Kadow T, Yurube T, Hartman R, Ngo K, Dong Q, Pohl P, Coelho JP, Kang J, Vo N and Sowa G: NSAID use in intervertebral disc degeneration: What are the effects on matrix homeostasis in vivo? Spine J 17: 1163-1170, 2017.

21. Daly C, Ghosh P, Jenkin G, Oehme D and Goldschlager T: A review of animal models of intervertebral disc degeneration: Pathophysiology, regeneration, and translation to the clinic. Biomed Res Int 2016: 5952165, 2016.

22. Liu H-T, Wang W-M, Lin Z-J, Chen G-X, Lin G-Y and Li P-S: Alterations in imaging and histopathology after aspiration of nucleus pulposus of rabbit lumbar intervertebral disc. J Clin Rehabil Tissue Eng Res 18: 1313-1318, 2014.

23. Upadhyay A, Senyschyn D, Santos L, Gu R, Carroll GJ and Jazayeri JA: K/BxN serum transfer arthritis is delayed and less severe in leukaemia inhibitory factor (LIF)-deficient mice. Clin Exp Immunol 169: 71-78, 2012.

24. Jiang Y, Xiao Q, Hu Z, Pu B, Shu J, Yang Q, Lao H and Hao J: Tissue levels of leukemia inhibitory factor vary by osteoarthritis grade. Orthopedics 37: e460-e464, 2014.

25. Adams MA and Roughley PJ: What is intervertebral disc degeneration, and what causes it? Spine (Phila Pa 1976) 31: 2151-2161, 2006. 
26. Videman T, Gibbons LE and Battié MC: Age-and pathology-specific measures of disc degeneration. Spine (Phila Pa 1976) 33: 2781-2788, 2008.

27. Terry DE, Chopra RK, Ovenden J and Anastassiades TP Differential use of Alcian blue and toluidine blue dyes for the quantification and isolation of anionic glycoconjugates from cell cultures: Application to proteoglycans and a highmolecular-weight glycoprotein synthesized by articular chondrocytes. Anal Biochem 285: 211-219, 2000.

28. Silacci P, Dayer JM, Desgeorges A, Peter R, Manueddu C and Guerne PA: Interleukin (IL)-6 and its soluble receptor induce TIMP-1 expression in synoviocytes and chondrocytes, and block IL-1-induced collagenolytic activity. J Biol Chem 273: 13625-13629, 1998.
29. Tilg H, Dinarello CA and Mier JW: IL-6 and APPs: Anti-inflammatory and immunosuppressive mediators. Immunol Today 18: 428-432, 1997.

30. Nemoto O, Yamada H, Mukaida M and Shimmei M: Stimulation of TIMP-1 production by oncostatin $\mathrm{M}$ in human articular cartilage. Arthritis Rheum 39: 560-566, 1996.

31. Metcalf D: Leukemia inhibitory factor-a puzzling polyfunctional regulator. Growth factors 7: 169-173, 1992.

32. Wilks AF: Two putative protein-tyrosine kinases identified by application of the polymerase chain reaction. Proc Natl Acad Sci USA 86: 1603-1607, 1989. 